\title{
Predictive Threat Assessment via Reachability Analysis and Set Invariance Theory
}

\author{
Paolo Falcone*, Mohammad Ali ${ }^{*, \dagger}$ and Jonas Sjöberg*
}

\begin{abstract}
We propose two model based threat assessment methods for semi-autonomous vehicles, i.e., human driven vehicles with autonomous driving capabilities. Based on information about the surrounding environment, we introduce a set of constraints on the vehicle states, which are satisfied under "safe" driving conditions. Then we formulate the threat assessment problem as a constraint satisfaction problem. Vehicle and driver mathematical models are used in order to predict future constraints violation, indicating the possibility of accident or loss of vehicle control, hence the need of assisting the driver. The two proposed methods differ in the models used to predict the vehicle motion within the surrounding environment.

We demonstrate the proposed methods in a roadway departure application and validate it through experimental data.
\end{abstract}

Index Terms-Active Safety, Semi-Autonomous Vehicles, Threat Assessment, Decision Making, Reachability Analysis, Invariant Set Theory.

\section{INTRODUCTION}

Classical active safety systems like, e.g., yaw stability control, only affect the dynamical behavior of the vehicle while its motion control within the environment is left to the driver. Thanks to recent advances in sensing technologies $[16,6]$, modern Advanced Driver Assistance Systems (ADAS) can instead influence both the dynamical behavior of the vehicle and its motion within the surrounding environment to prevent accidents. In particular, sensors measurements can be fused to obtain information about the surrounding environment like, e.g., road geometry and relative position and velocity of moving objects. This is demonstrated in, e.g., [12] where a standard radar, typically used for adaptive cruise control, is used along with an off the shelf camera for joint road geometry estimation and vehicle tracking.

Motivated by the described state-of-the-art in sensing technologies, the current trend in the development of ADAS for passenger cars points towards systems with increased autonomous driving capabilities in complex environments [23], beyond what is currently available in production active safety systems. In particular, future ADAS are envisioned to assist the driver in negotiating curves or intersections and autonomously drive the vehicle in order to avoid accidents, if needed. Prototypes of autonomous vehicles have been shown to successfully accomplish different and complex driving tasks at both low speeds, in urban environments, [26, 24, 3] and in high speed maneuvers on low friction surfaces $[13,14]$. The promising

\footnotetext{
*Department of Signals and Systems, Chalmers University of Technology, SE-41296 Göteborg, Sweden, E-mail: falcone@chalmers.se, jonas.sjoberg@chalmers.se

†Volvo Car Corporation, Active Safety \& Chassis, 96410/PV4A, 40531 Göteborg Sweden, E-mail: mali21@volvocars.com
}

results obtained with these prototypes of autonomous vehicles clearly demonstrate the possibilities offered by the autonomous driving technologies for active safety applications. However, in active safety applications for passengers cars it is essential that an assisting autonomous driving intervention is initiated if and only if it is needed, i.e., if a risk of accident is detected that the driver is not able to avoid. The formulation of transition conditions between, e.g., a manual and a fully autonomous driving mode, is not trivial.

In this paper, we consider a threat assessment problem for semi-autonomous vehicles, i.e., human driven vehicles with autonomous driving capabilities. We assume that information about the surrounding environment is given with a certain amount of preview and focus on the problem of determining, based on the current vehicle state, whether the driver needs assistance in order to safely (i.e., without colliding with other objects in the surrounding environment or losing stability) accomplish a driving task. Although the method is general and can be used in a wide range of accident scenarios, for the sake of easy illustration, in this paper we consider a lane departure application. In particular, we consider lane guidance systems assisting the driver in maintaining the vehicle within the lane boundaries.

In several lane guidance algorithms, the transition conditions to an autonomous driving mode are formulated based on the "Time to Line Crossing" (TLC). An intervention or warning is issued once the TLC passes a predefined threshold. An overview and assessment of methods for calculating the TLC is provided in [21]. Artificial potential fields, instead, are used in the method presented in [29], where lane crossings are prevented by introducing cost functions whose value increase as the vehicle approaches the lane boundaries. An optimization-based approach is presented in [2]. Every time step, based on current vehicle state and information about the surrounding environment, a Model Predictive Controller is used to compute a vehicle trajectory over a future time horizon. An assisting intervention is issued if the computed trajectory is considered hazardous. We observe that in these approaches the transition criteria activating the autonomous driving interventions are based on the evaluation of a controller behavior and/or the limitations of the vehicle capability of remaining stable and within the lane, without accounting for the limitations of the driver's ability to perform the same task. This might lead to the initiation of an autonomous driving intervention in situations where the driver does not need assistance. On the other hand, if the controller is outperforming the driver, no intervention might be issued at all. In this paper, instead, we propose two threat assessment methods 
explicitly accounting for the human driving behavior. The idea underlying the proposed methods is to use vehicle and driver mathematical prediction models, along with information about the surrounding environment, to assess the risk of accidents over a future, finite time horizon. We demonstrate the proposed method in a lane guidance application and validate it through experimental data. The paper is organized as follows. In Section II, we provide basic definitions and results on reachability analysis and set invariance theory. In Section III, we present the vehicle and driver modeling used next, in Section IV, where the threat assessment algorithms are presented. In Section $\mathrm{V}$, we discuss the validation results obtained through the proposed algorithms, while Section VI closes the paper with final remarks.

\section{BACKGROUND ON SET INVARIANCE THEORY AND REACHABILITY ANALYSIS}

In this section we introduce a few definitions and recall basic results on set invariance theory and reachability analysis for constrained systems. A comprehensive survey of papers on set invariance theory can be found in [9]. This section adopts the notation used in [15].

We will denote the set of all real numbers and positive integers by $\mathbb{R}$ and $\mathbb{N}^{+}$, respectively.

Denote by $f_{a}$ the state update function of an autonomous discrete time system

$$
x(t+1)=f_{a}(x(t), w(t)),
$$

where $x(t)$ and $w(t)$ denote the state and disturbance vectors, respectively. System (1) is subject to the constraints

$$
x(t) \in \mathcal{X} \subseteq \mathbb{R}^{n}, w(t) \in \mathcal{W} \subseteq \mathbb{R}^{d},
$$

where $\mathcal{X}$ and $\mathcal{W}$ are polyhedra that contain the origin in their interiors. For the autonomous system (1)-(2),

Definition 1 (Reachable set (for autonomous systems)): we define the one-step robust reachable set for initial states $x$ contained in the set $\mathcal{S}$ as

$$
\begin{aligned}
\operatorname{Reach}_{f_{a}}(\mathcal{S}, \mathcal{W}) & \triangleq\left\{x \in \mathbb{R}^{n} \mid\right. \\
& \left.\exists x(0) \in \mathcal{S}, \exists w \in \mathcal{W}: x=f_{a}(x(0), w)\right\} .
\end{aligned}
$$

For the nominal system, i.e., with $w(t)=0, \forall t$, the onestep reachable set is defined as

$$
\operatorname{Reach}_{f_{a}}(\mathcal{S}) \triangleq\left\{x \in \mathbb{R}^{n} \mid \exists x(0) \in \mathcal{S}: x=f_{a}(x(0))\right\} .
$$

For the autonomous system (1)-(2), we define the dual of the reachable set as,

Definition 2 (Pre set (for autonomous systems)): the set of states that evolves to $\mathcal{S}$ in one step, as

$$
\operatorname{Pre}_{f_{a}}(\mathcal{S}, \mathcal{W}) \triangleq\left\{x \in \mathbb{R}^{n} \mid f_{a}(x, w) \in \mathcal{S}, \forall w \in \mathcal{W}\right\}
$$

For the nominal system, the "Pre" set is defined as

$$
\operatorname{Pre}_{f_{a}}(\mathcal{S}) \triangleq\left\{x \in \mathbb{R}^{n} \mid f_{a}(x) \in \mathcal{S}\right\} .
$$

Equivalently, for the system with inputs

$$
x(t+1)=f(x(t), u(t), w(t)),
$$

subject to the constraints

$$
x(t) \in \mathcal{X}, u(t) \in \mathcal{U} \subseteq \mathbb{R}^{m}, w(t) \in \mathcal{W},
$$

Definition 3 (Reachable set (for systems with external inputs)): the one-step robust reachable set for initial states $x$ contained in the set $\mathcal{S}$ is defined as

$$
\begin{aligned}
\operatorname{Reach}_{f}(\mathcal{S}, \mathcal{W}) \triangleq\left\{x \in \mathbb{R}^{n} \mid\right. \\
\exists x(0) \in \mathcal{S}, \exists u \in \mathcal{U}, \exists w \in \mathcal{W}: x=f(x(0), u, w)\} .
\end{aligned}
$$

Moreover,

Definition 4 (Pre set (for systems with external inputs)): we define the set of states which can be driven into the target set $\mathcal{S}$ in one time step as

$$
\begin{aligned}
\operatorname{Pre}_{f}(\mathcal{S}, \mathcal{W}) & \triangleq\left\{x \in \mathbb{R}^{n} \mid\right. \\
& \exists u \in \mathcal{U} \text { s.t. } f(x, u, w) \in \mathcal{S}, \forall w \in \mathcal{W}\} .
\end{aligned}
$$

The sets $\operatorname{Reach}_{f}(\mathcal{S})$ and $\operatorname{Pre}_{f}(\mathcal{S})$, for the nominal system with external inputs, are defined similarly as in (4) and (6), respectively.

In this paper we will use robust invariant sets and robust control invariant sets. The following definitions are derived from $[9,8,7]$.

Definition 5 (Robust positive Invariant Set): A set $\mathcal{O}$ is said to be a positive invariant set for the autonomous system (1) subject to the constraints in (2), if

$$
x(0) \in \mathcal{O} \Rightarrow x(t) \in \mathcal{O}, \quad \forall t \in \mathbb{N}^{+}
$$

Definition 6 (Maximal Positive Invariant Set $\mathcal{O}_{\infty}$ ): The set $\mathcal{O}_{\infty}$ is the maximal invariant set of the autonomous system (1) subject to the constraints in (2), if $0 \in \mathcal{O}_{\infty}, \mathcal{O}_{\infty}$ is positive invariant and $\mathcal{O}_{\infty}$ contains all positive invariant sets that contain the origin.

Control invariant sets are defined for systems subject to external inputs. The following definitions are derived from $[9,8,7,19]$.

Definition 7 (Control Invariant Set): A set $\mathcal{C} \subseteq \mathcal{X}$ is said to be a control invariant set for the system in (7) subject to the constraints in $(8)$, if

$x(t) \in \mathcal{C} \Rightarrow \exists u(t) \in \mathcal{U}$ such that $f(x(t), u(t)) \in \mathcal{C}, \forall t \in \mathbb{N}^{+}$

Definition 8 (Maximal Control Invariant Set $\mathcal{C}_{\infty}$ ): The set $\mathcal{C}_{\infty}$ is said to be the maximal control invariant set for the system in (7) subject to the constraints in (8), if it is control invariant and contains all control invariant sets contained in $\mathcal{X}$.

\section{MODELING}

In this section, we present the vehicle and driver's steering behavior mathematical models used in Section IV as basis of the threat assessment algorithm. 


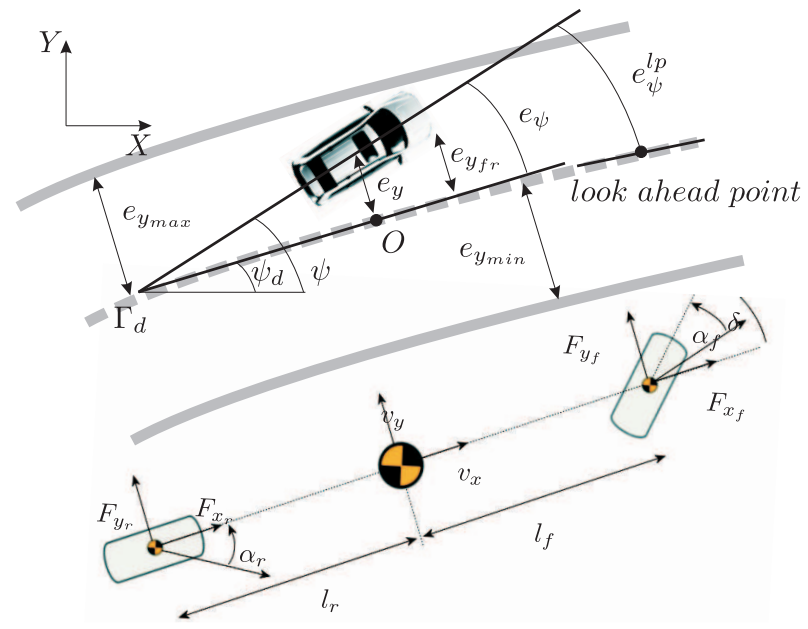

Fig. 1. Vehicle modeling notation.

\section{A. Vehicle model}

Consider the vehicle model sketched in Figure 1. The vehicle motion within the lane, subject to the lateral and yaw dynamics, is described by the following set of differential equations

$$
\begin{aligned}
m \dot{v}_{y} & =-m v_{x} \dot{\psi}+2\left[F_{y_{f}}+F_{y_{r}}\right], \\
J_{z} \ddot{\psi} & =2\left[l_{f} F_{y_{f}}-l_{r} F_{y_{r}}\right], \\
\dot{e}_{\psi} & =\dot{\psi}-\dot{\psi}_{d}, \\
\dot{e}_{y} & =v_{y}+v_{x} e_{\psi},
\end{aligned}
$$

where $m$ and $J_{z}$ denote the vehicle mass and yaw inertia, respectively, $l_{f}$ and $l_{r}$ are the distances of the vehicle center of gravity from the front and rear axles, respectively, $v_{x}$ and $v_{y}$ are the longitudinal and lateral velocities, respectively, in the vehicle body frame, $\dot{\psi}$ is the turning rate, where $\psi$ denotes the vehicle orientation w.r.t. the fixed global frame $(X, Y)$ in Figure 1. $F_{y_{f}}, F_{y_{r}}$ are the lateral tire forces at the front and rear axles, respectively. In (13c) and (13d), $e_{\psi}$ and $e_{y}$ denote the vehicle orientation and position errors, respectively, w.r.t. the road centerline and $\psi_{d}$ is the desired vehicle orientation, i.e., the slope of the tangent to the curve $\Gamma_{d}$ in the point $O$.

The lateral tire forces in (13a) and (13b) are generated at the tire contact patch and are, in general, nonlinear functions of the vehicle states. In this paper, we compute the lateral tire forces as

$$
F_{y i}=-C_{i} \alpha_{i}, i \in\{f, r\},
$$

where $C_{i}$ are the tire cornering stiffness coefficients at the two axles and $\alpha_{i}$ are the tyre slip angles which, for small values, can be approximated as

$$
\alpha_{f}=\frac{v_{y}+l_{f} \dot{\psi}}{v_{x}}-\delta, \quad \alpha_{r}=\frac{v_{y}-l_{r} \dot{\psi}}{v_{x}},
$$

where $\delta$ denotes the front steering angle as depicted in Figure 1.

Remark 1: The simplified linear tire model (14) well approximates more complex nonlinear tire characteristics [4] for small tire slip angles, i.e., $\alpha_{i} \in\left[\alpha_{i_{\min }}, \alpha_{i_{\max }}\right]$. However, this interval also corresponds to a "normal driving" region where drivers usually operate $[27,18]$.
For a given vehicle longitudinal speed $v_{x}$, the model (13)(15) can be compactly written as

$$
\dot{x}(t)=A x(t)+B u(t)+E w(t),
$$

where $x=\left[v_{y}, \dot{\psi}, e_{\psi}, e_{y}\right]^{T}$ and $w=\dot{\psi}_{d}$ are the state and the disturbance vectors and $u=\delta$ is the steering input command.

\section{B. Driver model}

In this section, we consider the vehicle model (16) and present a feedback control law resembling the human driver's steering behavior while performing a lane following task. The driver model, combined with the vehicle model (16), is used next in Section IV as basis of the proposed threat assessment algorithm. The literature on the modeling of driver steering is rich, early studies on driver modeling date back to the sixties and demonstrated the importance of preview information for human drivers [28]. McRuer et al. were among the first proposing a human driver's steering "preview control" algorithm consisting of a "pursuing" part and a closed loop correcting part [22]. The various preview control algorithms can be divided in two main groups, depending on whether the "preview signal" is provided as a reference or a disturbance [25]. In this paper, the driver's steering behavior is described by a preview control law, where the "preview signal" enters as a disturbance.

Define the orientation error $e_{\psi}^{l p}$, w.r.t. the look-ahead point in Figure 1, as

$$
e_{\psi}^{l p}=\psi-\psi_{d}^{l p}=e_{\psi}+\Delta \psi_{d},
$$

where $\psi_{d}^{l p}$ is the desired orientation at time $t+t_{l p}$, with $t$ the current time, $\Delta \psi_{d}=\psi_{d}-\psi_{d}^{l p}$ and $t_{l p}$ the preview time that can be mapped into the preview distance $d_{l p}$ under the assumption of constant speed $v_{x}$.

We consider the vehicle model (13) and compute the steering angle $\delta$ as

$$
\delta=K_{y} e_{y}+K_{\psi} e_{\psi}^{l p},
$$

with $K_{y}, K_{\psi}$ gains that are, in general, time varying and might be updated online.

Clearly, $\Delta \psi_{d}$ in (17) depends on the preview time $t_{l p}$ that, in our modeling framework, is considered as a parameter of the driver's model and can be identified from experimental data. Recursive least squares estimation results of the driver's model parameters are demonstrated in Section V.

\section{Driver controlled vehicle model}

We consider the autonomous system, obtained by combining the vehicle and driver models (16) and (18), respectively, that can be compactly written as

$$
\dot{x}_{a}(t)=A_{a} x_{a}(t)+E_{a} w_{a}(t),
$$

where $x_{a}=\left[v_{y}, \dot{\psi}, e_{\psi}, e_{y}\right]^{T}$ and $w_{a}=\left[\dot{\psi}_{d}, \Delta \psi_{d}\right]^{T}$ are the state and the disturbance vectors, respectively. The definitions of the matrices $A_{a}, E_{a}$ are straightforward. 


\section{System Constraints}

Next we define a set of operating conditions, in the space of the states and inputs of systems (16) and (19), corresponding to stable driving within the lane boundaries.

We denote by $e_{y_{i j}}, i \in\{f, r\}, j \in\{l, r\}$, the distances of the four vehicle corners from the lane centerline. By assuming small orientation errors, $e_{y_{i j}}$ can be written as

$$
\begin{array}{ll}
e_{y_{f l}}=e_{y}+\frac{c}{2}+a e_{\psi}, & e_{y_{f r}}=e_{y}-\frac{c}{2}+a e_{\psi}, \\
e_{y_{r l}}=e_{y}+\frac{c}{2}-b e_{\psi}, & e_{y_{r r}}=e_{y}-\frac{c}{2}-b e_{\psi},
\end{array}
$$

where $c$ is the vehicle width, $a$ and $b$ are the distances of the center of gravity from the front and rear vehicle bumpers, respectively. Furthermore, in order to avoid possible vehicle instability due to the effects of the tire nonlinearities (see Remark 1), the vehicle can be forced to operate in a region of the state space by limiting the tire slip angles $\alpha_{i}, i \in\{f, r\}$.

The constraints on the vehicle position and slip angles can then be compactly written as

$$
\begin{aligned}
-e_{y_{\max }} & \leq e_{y_{i j}} \leq e_{y_{\max }}, \\
\alpha_{i_{\min }} & \leq \alpha_{i} \leq \alpha_{i_{\max }}, i \in\{f, r\}, j \in\{l, r\},
\end{aligned}
$$

where $e_{y_{\max }}$ is the maximum distance of the vehicle corners from the lane centerline. The model (13)-(15), subject to constraints (21), describes the stable vehicle motion within the lane. In such operating conditions assuming small orientation errors is deemed reasonable.

The constraints (21) can be compactly rewritten for the system (16) as

$$
\left[\begin{array}{ll}
H_{x} & H_{u}
\end{array}\right]\left(\begin{array}{l}
x \\
u
\end{array}\right) \leq h,
$$

while for the system (19) we have

$$
H_{a} x_{a} \leq h_{a}, H_{a}=H_{x}+H_{u} K, \quad h_{a}=h-H_{u} K_{\psi} \Delta \psi_{d} .
$$

\section{THREAT ASSESSMENT VIA REACHABILITY ANALYSIS AND SET INVARIANCE THEORY}

In this section we propose two threat assessment methods based on the vehicle and driver modeling presented in Section III. The idea underlying the proposed methods is to first introduce a set of constraints on the vehicle state and input trajectories, describing a "safe" driving. For the specific lane guidance application considered in this paper, the constraints are set by the lane boundaries and the stability limits the vehicle should not exceed, i.e., the inequalities (21). Hence, constraints satisfaction implies that the vehicle is traveling within the lane boundaries in a stable operating region. The threat assessment problem is then reformulated as a constraints satisfaction problem over a future time horizon, solved through the predictive, model based methods described next.

Based on the vehicle and driver models, every time step we calculate a "safe set". This is the set of vehicle states at current time $t$ which, according to the considered vehicle and driver models, are guaranteed to evolve to a given final set in finite time while satisfying the constraints (21). The safe set is updated in receding horizon, as new information about the surrounding environment is available, e.g., the coming road curvature in our lane guidance application. Once the safe set is computed, a set membership test on the current vehicle state is performed to check whether the vehicle is in the safe set and decide whether the driver needs to be assisted.

We use the reachability analysis and set invariance theory definitions, given in Section II for linear systems, to calculate the safe sets. Similar ideas can be found in [1] and [5] in the automotive and aerospace fields, respectively. In [1], the authors propose a collision detection method in autonomous driving. In particular, a traffic scenario is considered where the path of an autonomous vehicle has to be planned in order to avoid collisions with other traffic participants, whose future trajectory is unknown. A safe planned path for the autonomous vehicle is required to not intersect the stochastic reachable sets computed for each traffic participant, i.e., the set of future positions possibly occupied by the other traffic participants. In [5], a safety analysis of an aircraft autoland system is developed based on the calculation of reachable sets. In the landing phase, the set of the aircraft configurations, evolving within a safe envelope to the set of acceptable states at touchdown, is calculated as a backward reachable set.

In this paper we propose two predictive and model based threat assessment methods. In particular, in the first approach the vehicle motion within the lane is described by the vehicle model (16) only, while in the second we assume that the steering angle $\delta$ in (16) is generated by the state feedback control law (18) and consider the driver controlled vehicle model (19). Next in this section we will highlight how the difference in the used models reflects on the computational scheme used to compute the safe sets (Remark 4) and the results of the threat assessment (Remark 3).

In both approaches, the road curvature is assumed to be known over a future time horizon and to lie within a given set. In particular, we introduce the following assumptions on the disturbance signals $w$ and $w_{a}$ in (16) and (19), respectively.

Assumption 1: $w(t) \in \mathcal{W}, w_{a}(t) \in \mathcal{W}_{a}, \forall t \geq 0$, where $\mathcal{W} \subseteq \mathbb{R}, \mathcal{W}_{a} \subseteq \mathbb{R}^{2}$ are polyhedrons that contain the origin in their interiors.

We discretize the model (16) with a sampling time $T_{s}$, to obtain the following discrete time constrained system with disturbances

$$
\begin{array}{ll}
x(t+1)= & A^{d} x(t)+B^{d} u(t)+E^{d} w(t) \\
\text { subj. to } & {\left[\begin{array}{ll}
H_{x} & H_{u}
\end{array}\right]\left(\begin{array}{l}
x(t) \\
u(t)
\end{array}\right) \leq h}
\end{array}
$$

where, for the sake of simple notation, we have denoted the state, the disturbance and the time index with the same symbols as in the continuous time model (16).

Assumption 2: Every time instant $t$, the disturbances $w(t), w_{a}(t)$ are known over a finite time horizon of $N$ steps.

We recall that, every time instant $t$, the second component of the disturbance vector $w_{a}$, i.e., $\Delta \psi_{d}$, is based on the desired orientation $\psi_{d}^{l p}$ at time $t+t_{l p} / T_{s}$. This is the desired vehicle orientation at the look-ahead point. Hence, the Assumption 2 
on the disturbance $w_{a}$ requires the knowledge of the road geometry over a future time horizon $t_{l p} / T_{s}+N$. We define the set of admissible vehicle states as

$$
\mathcal{X}_{\text {feas }}=\left\{x \in \mathbb{R}^{4}:\left[\begin{array}{ll}
H_{x} & H_{u}
\end{array}\right]\left(\begin{array}{l}
x \\
u
\end{array}\right) \leq h\right\} .
$$

Every time instant, we consider a terminal target set $\mathcal{T} \subseteq$ $\mathcal{X}_{\text {feas. }}$. Further details about the choice of $\mathcal{T}$ are provided next, in Section IV-A. Moreover, denote by $W_{t}=$ $\left[w_{t}, w_{t+1}, \ldots, w_{t+N-1}\right]$, the sequence of disturbance samples over the time horizon $[t, t+N-1]$ and by $W_{t, i}=$ $\left[w_{t+i}, \ldots, w_{t+N-1}\right]$ any sequence extracted from $W_{t}$. We compute the sequence of states sets $X_{t}\left(W_{t}\right)=$ $\left[\mathcal{X}_{t}, \mathcal{X}_{t+1}, \ldots, \mathcal{X}_{t+N-1}\right]$ as:

$$
\begin{aligned}
\mathcal{X}_{t+i}\left(W_{t, i}\right) & =\mathcal{X}_{\text {feas }} \bigcap \operatorname{Pre}_{f}\left(\mathcal{X}_{t+i+1}, w_{t+i}\right), \\
& i=N-1, \ldots, 0, \\
\mathcal{X}_{t+N} & =\mathcal{T},
\end{aligned}
$$

where, $f$ denotes the right hand side of (24a). We call the set $\mathcal{X}_{t}$ the safe set at time $t$.

The calculation of the sequence $X_{t}\left(W_{t}\right)$ is performed every time step, based on the updated disturbance sequence $W_{t}$. In the set operator Pre. $(\cdot, \cdot)$ in (26), with a slight abuse of notation, a vector is used as second argument instead of a set. This corresponds to the case of known disturbance.

In summary, the proposed threat assessment algorithm is made of three main steps performed every time instant

1) select the terminal target set $\mathcal{T}$,

2) based on the future disturbance sequence $W_{t}$ and the set $\mathcal{T}$, perform the backward calculation of the sequence of safe sets $\mathcal{X}_{t+i}$ according to (26),

3 ) check whether the current state $x(t)$ belongs to the safe set $\mathcal{X}_{t}$, in order to assess the driver's ability of safely driving the vehicle from the current state to the target set $\mathcal{T}$ over the future horizon of $N$ steps.

The steps of the method are detailed next in Algorithm 1. By construction, if the state of the system (24) at the current time $t$ belongs to the safe set $\mathcal{X}_{t}$ (Step 10 of Algorithm 1), a steering controller exists such that the vehicle can be driven over the next $N$ time steps, while operating within its stability limits and without leaving the lane. That is, over the future $N$ time steps, the vehicle can be driven within the lane boundaries, while operating in a region of the system states and inputs space where the driver is deemed capable of driving without loosing vehicle stability (see Remark 1).

Algorithm 1 is based on the vehicle model (16). Next we propose a method based on the driver controlled vehicle model (19). In this case, the calculation of the safe sets is based on the reachable and invariant sets for autonomous systems, defined in Section II.
Algorithm 1: Input: Current state $x(t)$, target set $\mathcal{T}$, sequence of disturbances $W_{t}$, state update mapping $f=$ $\left(A^{d}, B^{d}, E^{d}\right)$, the constraints matrices $\left(H_{x}, H_{u}, h\right)$

Output: The safe set $\mathcal{X}_{t}$ at the current time $t$, safe flag Safe

1 let $\mathcal{X}_{t+N}=\mathcal{T}$,

2 for $i=N-1$ to 0

3 let $\mathcal{X}_{t+i+1}=\left\{x \in \mathbb{R}^{4}: H_{i+1} x \leq h_{i+1}\right\}$,

$4 \quad \operatorname{Pre}_{f}\left(\mathcal{X}_{t+i+1}, w_{t+i}\right)=\left\{x \in \mathbb{R}^{4}: \exists u \in \mathbb{R}\right.$ subj. to

$$
\begin{aligned}
& {\left[\begin{array}{ll}
H_{i+1} A^{d} & H_{i+1} B^{d}
\end{array}\right]\left(\begin{array}{c}
x \\
u
\end{array}\right) \leq} \\
& \left.\left[\begin{array}{l}
h_{i+1}-H_{i+1} E^{d} w_{t+i}
\end{array}\right]\right\},
\end{aligned}
$$

5 if $\operatorname{Pre}_{f}\left(\mathcal{X}_{t+i+1}, w_{t+i}\right)=\emptyset$ then $S a f e=0$, EXIT

6 else let $\operatorname{Pre}_{f}\left(\mathcal{X}_{t+i+1}, w_{t+i}\right)=$ $\left\{x \in \mathbb{R}^{4}: H_{\text {Pre }} x \leq h_{\text {Pre }}\right\}$

$7 \quad \mathcal{X}_{t+i}\left(W_{t, i}\right)=\left\{x \in \mathbb{R}^{4}:\right.$

$\left.\left[\begin{array}{cc}H_{\text {Pre }} & \mathbf{0} \\ H_{x} & H_{u}\end{array}\right]\left(\begin{array}{l}x \\ u\end{array}\right) \leq\left[\begin{array}{c}h_{\text {Pre }} \\ h\end{array}\right]\right\}$, end

$8 \quad$ if $\mathcal{X}_{t+i}\left(W_{t, i}\right)=\emptyset$ then $S a f e=0$, EXIT, end

9 end

10 if $x(t) \in \mathcal{X}_{t}$ then Safe $=1$,

11 else $S a f e=0$, end

12 EXIT.

We discretize the model (19) with a sampling time $T_{s}$, to obtain the following discrete time constrained autonomous system with disturbances

$$
\begin{array}{cl}
x_{a}(t+1)= & A_{a}^{d} x_{a}(t)+E_{a}^{d} w_{a}(t) \\
\text { subj. to } \quad & H_{a} x_{a}(t) \leq h_{a},
\end{array}
$$

where, for the sake of simple notation, we have again denoted the state, the disturbance and the time index with the same symbols as in (19). With a slight abuse of notation we let $\mathcal{X}_{\text {feas }}, \mathcal{X}_{t}$ and $\mathcal{T}$ denote the set of admissible states, the safe set at time $t$ and the terminal set, respectively, also for the system (27). For the system (27), the set of admissible states is defined as

$$
\mathcal{X}_{\text {feas }}=\left\{x \in \mathbb{R}^{4}: H_{a} x \leq h_{a}\right\} .
$$

The sequence of safe sets for the constrained system (27) is then computed through (26), where the mapping $f$ is replaced by the mapping $f_{a}$, denoting the right hand side of (27a) and the disturbance vectors $w_{i}$ are replaced by $w_{a_{i}}$.

The steps of the threat assessment based on the vehicle and driver's models presented in Sections III-A and III-B, respectively, are detailed in Algorithm 2.

Remark 2: If the safe set $\mathcal{X}_{t}$ is empty (Steps 5, 7 of Algorithm 2) or if the current state $x_{a}(t)$ does not belong to the safe set $\mathcal{X}_{t}$ (Steps 9, 10 of Algorithm 2), there exists no state trajectory of system (16) that, under the driver's steering feedback control law (18), can evolve from the current state $x_{a}(t)$ to the target set $\mathcal{T}$, while satisfying the constraints (21). On the other hand, a different, in general time varying, steering law might exist driving the vehicle from the current state $x_{a}(t)$ to the target set, while satisfying the constraints (21). The existence of such steering law can be verified through Algorithm 1. 
Algorithm 2: Input: Current state $x_{a}(t)$, target set $\mathcal{T}$, sequence of disturbances $W_{t}$, state update mapping $f_{a}=\left(A_{a}^{d}, E_{a}^{d}\right)$, the constraints matrices $\left(H_{a}, h_{a}\right)$

Output: The safe set $\mathcal{X}_{t}$ at the current time $t$, safe flag Safe

1 let $\mathcal{X}_{t+N}=\mathcal{T}$,

2 for $i=N-1$ to 0

3 let $\mathcal{X}_{t+i+1}=\left\{x_{a} \in \mathbb{R}^{4}: H_{i+1} x_{a} \leq h_{i+1}\right\}$,

$4 \quad \operatorname{Pre}_{f_{a}}\left(\mathcal{X}_{t+i+1}, w_{t+i}\right)=\left\{x_{a} \in \mathbb{R}^{4}\right.$ : $\left.H_{i+1} A_{a}^{d} x_{a} \leq h_{i+1}-H_{i+1} E_{a}^{d} w_{t+i}\right\}$

5 if $\operatorname{Pre}_{f_{a}}\left(\mathcal{X}_{t+i+1}, w_{t+i}\right)=\emptyset$ then $S a f e=0$, EXIT

$6 \quad$ else $\mathcal{X}_{t+i}\left(W_{t, i}\right)=\left\{x_{a} \in \mathbb{R}^{4}\right.$ :

$\left.\left[\begin{array}{c}H_{i+1} A_{a}^{d} \\ H_{a}\end{array}\right] x_{a} \leq\left[\begin{array}{c}h_{i+1}-H_{i+1} E_{a}^{d} w_{t+i} \\ h_{a}\end{array}\right]\right\}$, end

7 if $\mathcal{X}_{t+i}\left(W_{t, i}\right)=\emptyset$ then $S a f e=0$, EXIT, end

8 end

9 if $x_{a}(t) \in \mathcal{X}_{t}$ then Safe $=1$,

10 else $S a f e=0$, end

11 EXIT.

Remark 3: In Algorithm 2, the restriction of the steering

law to the class of linear state feedback control laws (18) leads to smaller safe sets than in Algorithm 1.

The oversimplified driver model (18) might not be able to capture the driver's steering behavior in hazardous scenarios like, e.g., when $(i)$ he/she is required to drive beyond the vehicle stability limits or (ii) he/she is distracted or drowsy. Nevertheless, for the threat assessment problem formulation considered in this paper, we believe it is not necessary to exhaustively describe the driver's behavior. In fact, if the vehicle is either beyond its stability limits or the lane boundaries, case- $(i)$, a threat assessment problem is not meaningful any longer and an assisting intervention has to be issued. In case(ii), since our threat assessment problem formulation is not depending on any driver monitoring system, driver's distraction or drowsiness is not accounted for. Hence an assisting intervention would not be issued as long as the vehicle can be maintained within its stability limits and the lane boundaries by the considered "nominal" driver (i.e., behaving according to model (18)). However, the vehicle, driven by the drowsy or distracted driver, would very likely eventually exit the "safe set", calculated based on the "nominal" driver behavior, thus enabling the activation of a lower level intervention.

Remark 4: We observe that Steps 4 and 7 of Algorithm 1 involve a projection operation, while Algorithm 2 does not. In general, the projection operation can be quite involving depending on the dimension of the state and input spaces.

In the proposed approaches we formulate the threat assessment problem as a constraint satisfaction problem, for which efficient methods [10] exist. In particular, we calculate a polyhedral representations of the safe sets rather than just assessing the constraint satisfaction for the current vehicle state and the road curvature over the prediction horizon. A polyhedral representation of the safe set can be exploited for both control and verification purposes.

As last remark of this section, we observe that the Algorithm 2 assesses the capability of a driver, whose steering behavior is modeled by (18), of driving the vehicle, modeled by (16), from the current state $x_{a}(t)$ to the target set $\mathcal{T}$.

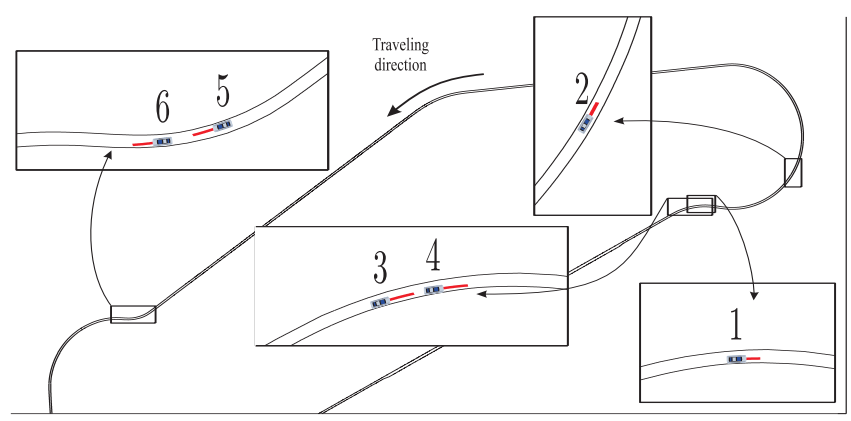

Fig. 2. Test track used for the experimental validation. Next, validation results of the Algorithms 1 and 2 are shown when the vehicle is in the marked positions.

Hence, just like as in Algorithm 1, constraint satisfaction is not guaranteed after the time $t+N$. In the next section we comment the choice of the target set $\mathcal{T}$ and propose a method for guaranteing persistent constraint satisfaction, i.e., that the driver will maintain the vehicle within $\mathcal{T}$, for $t>t+N$.

\section{A. Terminal Set}

The choice of the terminal set $\mathcal{T}$ in the threat assessment Algorithms 1 and 2 affects the effectiveness and the conservativeness of the algorithms. Indeed, the simplest choice is setting $\mathcal{T}=\mathcal{X}_{\text {feas }}$. In this case, Algorithms 1 and 2 can be used to assess the driver's ability of driving safely only over the future $N$ time steps. As alternative, for Algorithm 1, the set $\mathcal{T}$ could be chosen as $\mathcal{T}=\mathcal{C}_{\infty}$, where $\mathcal{C}_{\infty} \subseteq \mathcal{X}_{\text {feas }}$ is the maximal robust control invariant set for the constrained system with inputs (24). We recall that in this case,

$x(t+N) \in \mathcal{C}_{\infty} \Rightarrow x(t+N+k) \in \mathcal{C}_{\infty}, \forall w(t) \in \mathcal{W}, k \in \mathbb{N}^{+}$,

that is, the vehicle will be kept within the lane and its stability limits, despite all admissible lane curvature beyond the lookahead point. Similarly, for Algorithm 2, the set $\mathcal{T}$ could be chosen as $\mathcal{T}=\mathcal{O}_{\infty}$, where $\mathcal{O}_{\infty} \subseteq \mathcal{X}_{\text {feas }}$ is the maximal robust positive invariant set for the constrained autonomous system (27). In this case,

$x_{a}(t+N) \in \mathcal{O}_{\infty} \Rightarrow x_{a}(t+N+k) \in \mathcal{O}_{\infty}, \forall w_{a}(t) \in \mathcal{W}_{a}$,

that is, the driver is deemed capable of keeping the vehicle within the lane and its stability limits, despite all admissible lane curvature beyond the look-ahead point. Nevertheless, setting the final set equal to the maximal robust control invariant set or the maximal robust positive invariant set for Algorithms 1 and 2, respectively, might lead to high conservativeness of the threat assessment algorithms.

\section{RESULTS}

The algorithms presented in Section IV have been experimentally validated and the results are shown next in this section. Data have been logged by driving a Volvo V50 along the test track shown in Figure 2. This track is narrow in several sections and has many sharp curves, thus resembling a country road. The logged data has then been offline post-processed through the Algorithms 1 and 2 in order to calculate the safe sets along the track. For the sake of brevity, the computed safe sets will be shown only for the vehicle positions along 
the track, marked in Figure 2 with numbers from 1 to 6 , where the vehicle either approaches or travel along a curve.

Data has been collected in order to calculate the state and the disturbances variables of the vehicle models (16) and (19). In particular the vehicle lateral velocity $v_{y}$ and yaw rate $\dot{\psi}$ have been measured through a high precision Inertial Measurement Unit (IMU). The vehicle position and orientation errors within the lane $e_{y}$ and $e_{\psi}$, respectively, and the desired orientation at the look-ahead point $\psi_{d}^{l p}$ are calculated through dual antennas GPS measurements and a digital map including the road geometry.

Remark 5: The set of measurements used in the proposed threat assessment methods could also be obtained on-line by using the measurements setup and the sensor fusion algorithms used in $[16,6,12]$ (see Section I). Nevertheless, depending on the combination of sensors set and sensors fusion algorithms, a lower accuracy set of measurements might be obtained compared to the data used in this paper for the validation of the proposed threat assessment algorithms.

We first present validation results of the driver model (18). The data used for parameters estimation have been collected in both normal and slightly rougher driving styles. Compared to the normal driving case, where the driver has been asked to drive according to the suggested road speed limits, in the rough driving case the driver was asked to drive as fast as possible. The problem of estimating the parameters $K_{y}, K_{\psi}$ and $t_{l p}$ in (18) has been formulated as a recursive nonlinear least squares problem since the driver model is linear only in the parameters $K_{y}, K_{\psi}$. The recursive nonlinear least squares method described in [17] has been used.

Figure 3(a) shows the identification results in normal driving conditions. We observe high uncertainty in the estimated parameters at the beginning of the considered time interval. The uncertainty is however reduced as soon as the vehicle enters a curve and the system is excited. Moreover, we observe that the estimated value of the parameter $K_{y}$ is very small and has a quite large variance. This indicates that the vehicle lateral deviation from the lane centerline $e_{y}$ has a minor or no influence on the steering angle. The obtained results are in line with the conclusions of previous studies [28, 22] and show that the human steering behavior is preeminently based on a pursuit component, i.e., based on the preview of the desired path. Figure 3(b), instead, shows the driver's model parameters estimation in rougher driving maneuvers. Due to higher excitation, the initial uncertainty in the estimated parameters is reduced quicker than in Figure 3(a). The estimated parameter $K_{y}$ is in this case slightly negative. We also observe a higher magnitude of the estimated parameter $K_{\psi}$ and a shorter look ahead time $t_{l p}$, compared to the normal driving case. We conclude that the correcting part is more relevant in rough than in normal driving. The driver model (18) has been used to validate, through experimental data, Algorithm 2.

Algorithms 1 and 2 have been implemented by setting $\mathcal{T}=$ $\mathcal{X}_{\text {feas }}$ as terminal set and using the following parameters

$$
\begin{gathered}
\alpha_{f_{\max }}=\alpha_{r_{\max }}=-\alpha_{f_{\min }}=-\alpha_{r_{\min }}=4^{\circ}, \\
e_{y_{\max }}=1.56 \mathrm{~m}, \quad N=35, \quad T_{s}=0.01 \mathrm{~s} .
\end{gathered}
$$

The vehicle parameters are shown in Table I.
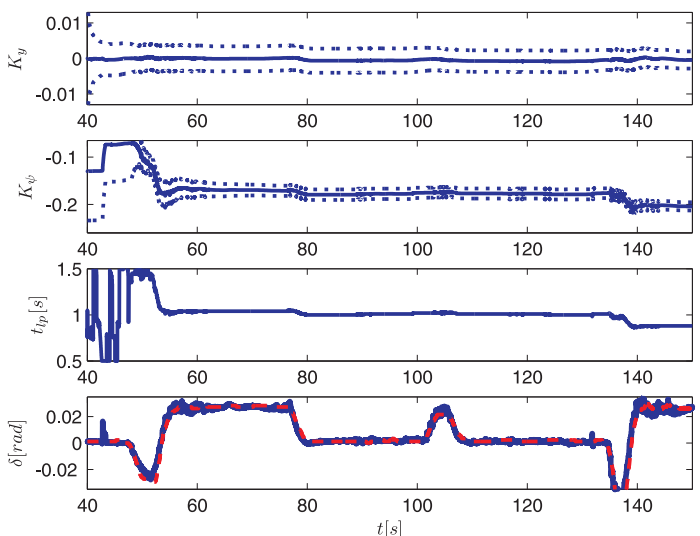

(a) Normal driving

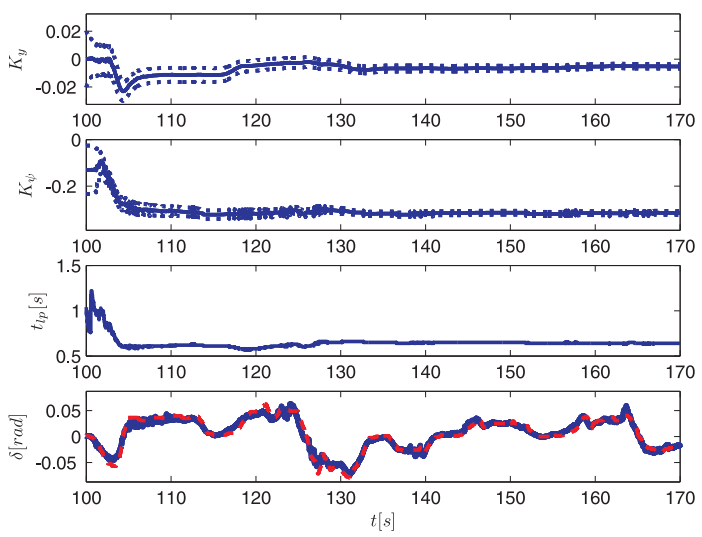

(b) Rough driving

Fig. 3. Recursive driver model parameters estimation. In the two upper plots of figures a) and b), the solid lines show the estimated parameters mean values while the dotted lines show their estimated variances. In the lower plots, the solid and dashed lines show the measured and predicted steering angles, respectively.

TABLE I

\begin{tabular}{|c|c|c|c|c|}
\hline$m$ & $J_{z}$ & $l_{f}$ & $l_{r}$ & $a$ \\
\hline $1695 \mathrm{~kg}$ & $2617 \mathrm{kgm}^{2}$ & $1.14 \mathrm{~m}$ & $1.50 \mathrm{~m}$ & $1.83 \mathrm{~m}$ \\
\hline$C_{f}$ & $C_{r}$ & $b$ & $c$ & \\
\hline $54 \mathrm{kNm} / \mathrm{rad}$ & $45 \mathrm{kNm} / \mathrm{rad}$ & $2.69 \mathrm{~m}$ & $1.77 \mathrm{~m}$ & \\
\hline
\end{tabular}

Remark 6: The calculation of the safe sets shown in this section has been implemented using the sets operations implemented in the Multiparametric Programming Toolbox (MPT) [20]. For the safe sets calculation, these operations require 1-2 minutes for Algorithm 1 and 0,3-0,4 seconds for Algorithm 2 on a laptop computer.

Consider the driving scenarios 1 and 2 shown in Figure 2 where the driver is negotiating a curve at a speed of $63 \mathrm{~km} / \mathrm{h}$, which can be considered a maneuver requiring normal driving skills. Denote by $t_{1}$ and $t_{2}$ the time instants, when the vehicle is in positions 1 and 2 on the track, respectively. Figure 4 shows cuts of the safe sets $\mathcal{X}_{t_{1}}$ and $\mathcal{X}_{t_{2}}$ calculated through both algorithms at times $t_{1}$ and $t_{2}$, respectively.

The following compact notation is introduced to denote sets cuts:

$\mathcal{X}_{t}^{3,4}(\tau)=\mathcal{X}_{t} \bigcap\left\{x \in \mathbb{R}^{4}:\left[\begin{array}{llll}1 & 0 & 0 & 0 \\ 0 & 1 & 0 & 0\end{array}\right] x=\left[\begin{array}{c}x^{1}(\tau) \\ x^{2}(\tau)\end{array}\right]\right\}$,

where the superscript $i$ in $x^{i}(\tau)$ denote the $i$-th component of the vector $x(\tau)$. The symbol $\mathcal{X}_{t}^{3,4}(\tau)$ will here denote a 


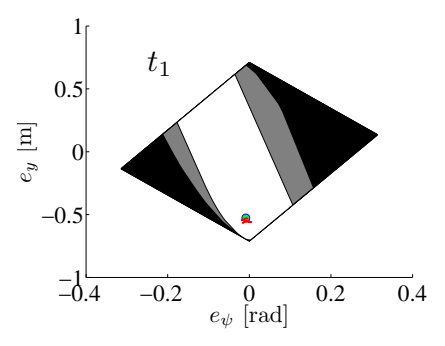

(a)

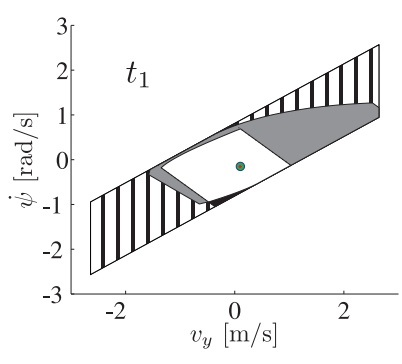

(b)

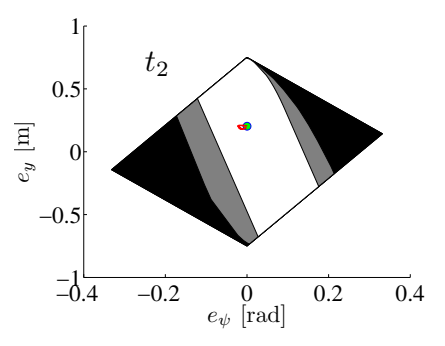

(c)

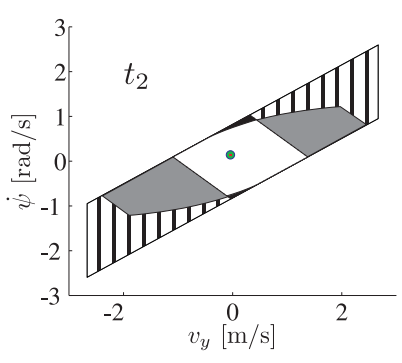

(d)

Fig. 4. Illustration of safe sets calculated through Algorithms 1 and 2 at times $t_{1}$ and $t_{2}$. The circles denote the measured current state of the vehicle at time $t_{1}$ while the solid lines denote the measured trajectory over the future horizon of $N$ steps. The sets filled with black and white stripes and the sets filled with solid black illustrate cuts of the feasibility sets $\mathcal{T}$ in Algorithms 1 and 2, respectively. The solid grey and white sets show cuts of the safe sets $\mathcal{X}_{t}$ calculated through Algorithm 1 and 2, respectively.

set in the $e_{y}-e_{\psi}$ space obtained by cutting $\mathcal{X}_{t}$ at $x^{1}(\tau)$ and $x^{2}(\tau)$, i.e. $v_{y}(\tau)$ and $\dot{\psi}(\tau)$. We observe that, as indicated in Remark 3, the feasibility and safe sets in Algorithm 2 are always enclosed by the corresponding sets in Algorithm 1. This can be explained by recalling that the feasibility and safe sets are derived from the constraints (21) which have been used in both algorithms. Moreover the inclusion of the driver model (18) in Algorithm 2 results in an additional set of equality constraints which further limits the set of admissible states.

We also note that in Figures 4(a), 4(c) the feasibility sets used in the two algorithms overlap. The additional limitations enforced by the driver model (18) only affects the terminal constraints that directly depend on the steering input $\delta$, which is not the case for $e_{y}$ or $e_{\psi}$.

The states $x\left(t_{1}\right)$ and $x\left(t_{2}\right)$ are marked with a circle in Figure 4. We observe that, for Algorithm 1, $x\left(t_{1}\right) \in$ $\mathcal{X}_{t_{1}}^{3,4}\left(t_{1}\right), x\left(t_{1}\right) \in \mathcal{X}_{t_{1}}^{1,2}\left(t_{1}\right)$ and $x\left(t_{2}\right) \in \mathcal{X}_{t_{2}}^{3,4}\left(t_{2}\right), x\left(t_{2}\right) \in$ $\mathcal{X}_{t_{2}}^{1,2}\left(t_{2}\right)$. Hence, from both the initial states $x\left(t_{1}\right), x\left(t_{2}\right)$, the vehicle is predicted to safely travel over a horizon of $N$ steps. This is confirmed by the vehicle state trajectories measured over the time intervals $\left[t, t+N T_{s}\right], t=\left\{t_{1}, t_{2}\right\}$ and reported in Figure 4 with solid lines. In particular, starting from the initial states $x\left(t_{1}\right)$ and $x\left(t_{2}\right)$, the measured vehicle state trajectories entirely evolve over the next $N$ steps within the sets $\mathcal{T}_{1}$ and $\mathcal{T}_{2}$, respectively, where $\mathcal{T}_{1}=\bigcup_{t=t_{1}}^{t_{1}+N-1} \mathcal{T}^{3,4}(t), \mathcal{T}_{2}$ is similarly defined and the sets $\mathcal{T}^{3,4}$ are obtained by replacing $\mathcal{X}_{t}$ with $\mathcal{T}$ in (29), i.e., the vehicle "safely" travels over the time intervals $\left[t, t+N T_{s}\right], t=\left\{t_{1}, t_{2}\right\}$, as predicted through Algorithm 1. In Figure 4, we also observe that $x_{a}\left(t_{1}\right) \in \mathcal{X}_{t_{1}}$ and $x_{a}\left(t_{2}\right) \in \mathcal{X}_{t_{2}}$ when the safe sets are calculated using Algorithm 2. Recall that, for the sake of simple readability, we have adopted the same notation for the safe sets obtained with the two algorithms.

Figure 5 shows the safe sets $\mathcal{X}_{t_{3}}$ and $\mathcal{X}_{t_{4}}$ corresponding to the positions 3 and 4 of the track in Figure 2, when the driver is negotiating a curve at a speed of approximately $92 \mathrm{~km} / \mathrm{h}$, traveling close to the lane edge. We observe that, for Algorithm 1, $x\left(t_{3}\right) \in \mathcal{X}_{t_{3}}$, while $x\left(t_{4}\right) \notin \mathcal{X}_{t_{4}}$. That is, the vehicle is predicted to safely travel over the time interval $\left[t_{3}, t_{3}+N T_{s}\right]$, while a constraint violation is predicted over the time interval $\left[t_{4}, t_{4}+N T_{s}\right]$. This is confirmed by the actual vehicle trajectories shown by the solid lines. Similar results are obtained through Algorithm 2 and also shown in Figure 5. We observe that, starting from the position 4, the driver cuts the curve in order to quickly traverse the path. However, such driving behavior might not be considered unsafe and an intervention should be avoided. Methods for suppressing warnings or interventions have even been proposed in [11] for a lane departure warning system. We observe that, in the framework proposed in this paper, instead, warnings or interventions in such driving situations could be avoided by just increasing the bounds $e_{y_{\max }}, e_{y_{\min }}$, for the "inner curve" to allow the driver cutting a curve without causing an intervention.

We finally consider the vehicle positions 5 and 6 in Figure 2, where the driver approaches the curve, with a velocity of approximately $84 \mathrm{~km} / \mathrm{h}$, which is a very high speed for such a narrow curve. We start with the results obtained with Algorithm 1. In Figure 6 we observe that $x\left(t_{5}\right) \in \mathcal{X}_{t_{5}}$ and $x\left(t_{6}\right) \in \mathcal{X}_{t_{6}}$, which means that there exists a control law that is capable of steering the vehicle $N$ time steps without violating constraints starting from both positions 5 and 6 . The safe set obtained with Algorithm 2, instead, is smaller, compared to Algorithm 1, and do not enclose the state $x_{a}\left(t_{6}\right)$. Hence both algorithms predict a safe driving when the vehicle starts from the position 5, while the results provided by the two algorithms significantly differ in position 6. In this case, Algorithm 2 correctly predicts a constraint violation. In this extreme driving scenario, according to Algorithm 2, the "nominal" driver is not capable of keeping the vehicle in a safe operating region. Depending on his or her driving skills, the actual driver, instead, might succeed in keeping the vehicle in such an operating region by deviating from the "nominal" behavior described by the model (18). Nevertheless, since a deviation from the nominal steering behavior is required, the situation can be considered critical and an intervention motivated.

Remark 7: As anticipated in Remark 2, Algorithm 1 showed that in position 6 , even if the driver is expected to violate constraints, there exists $\mathrm{a}$, in general time varying, control law that is capable of driving the vehicle over the future $N$ time steps, without violating constraints. We conclude that a steering controller could in this case be used to assist the driver. The combination of the driver's steering command and of such a low level steering controller could thus enlarge or move the safe set to enclose the state $x\left(t_{6}\right)$.

\section{CONCLUSION AND FUTURE WORKS}

We have presented two model based threat assessment methods for semi-autonomous vehicles and validated them in 


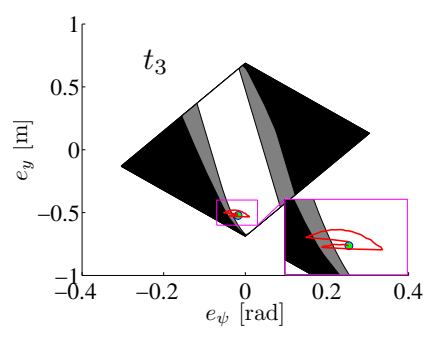

(a)

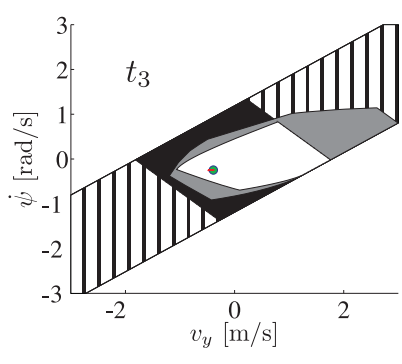

(b)

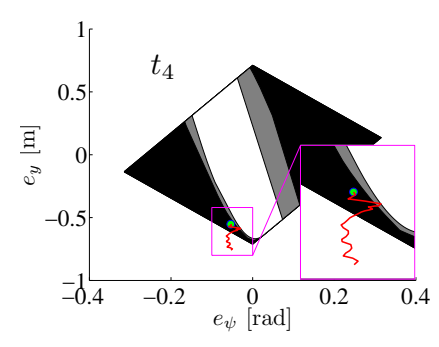

(c)

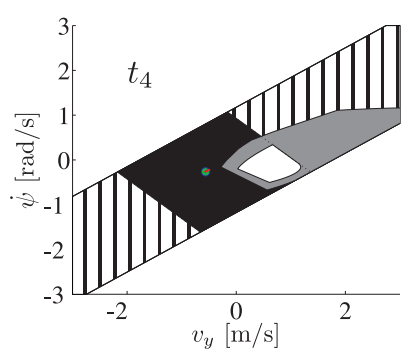

(d)

Fig. 5. Illustration of safe sets calculated through Algorithms 1 and 2 at times $t_{3}$ and $t_{4}$. The color convention in Figure 4 has been used.

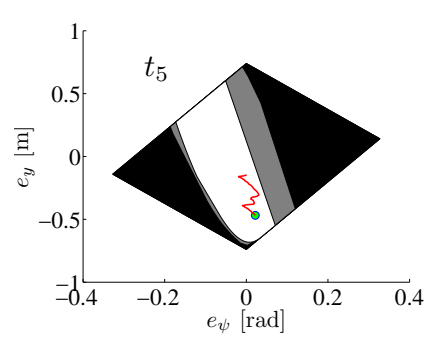

(a)

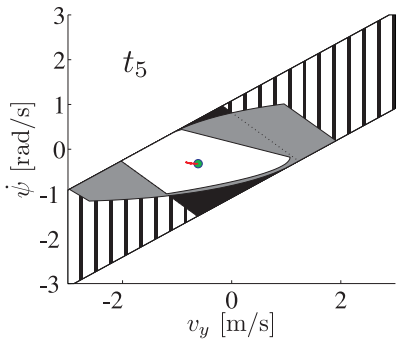

(b)

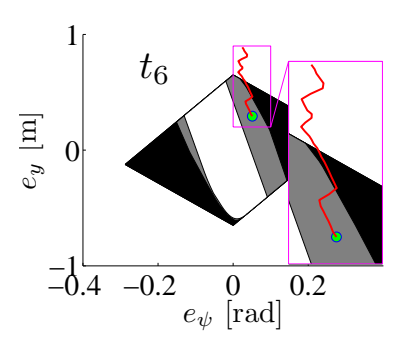

(c)

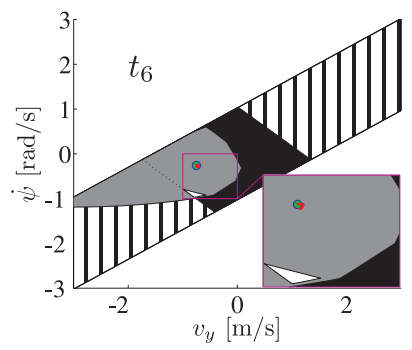

(d)

Fig. 6. Illustration of safe sets calculated through Algorithms 1 and 2 at times $t_{5}$ and $t_{6}$. The color convention in Figure 4 has been used.

a lane guidance application. The two approaches are based on reachability analysis tools and set invariance theory, and differ in the model used to predict the vehicle motion within the lane. In particular, in the second algorithm, the driver's steering behavior is estimated based on the road geometry and the vehicle state and used to predict the vehicle motion within the lane. The two methods have been validated offline by using experimental data. The obtained results demonstrate that the proposed methods can effectively predict lane crossing and vehicle instability over a future, finite time horizon, thus allowing the activation of driver assistance systems.

Nevertheless, we point out that (i) lower accuracy data might be available in an online implementation where a different measurements setup is used (see Remark 5), (ii) the proposed algorithms have been tested in a small range of operating conditions (iii) the adopted driver models might not be able to describe the human driver's driving behavior well, thus introducing a high level of uncertainty, (iv) the computational burden of the driver's model parameters identification and the safe sets calculation might represent an obstacle to the realtime implementation of the proposed algorithms. The points (i)-(iii) address robustness issues with respect to measurements errors and models uncertainties, while the point (iv) addresses computational complexity issues. The preliminary results presented in this manuscript motivate further investigations aiming at analyzing the impact of measurements errors and model uncertainties on the performance of the proposed threat assessment methods and proposing approaches in order to compensate for them. Moreover, computational complexity issues will be addressed by adopting real-time oriented code (see Remark 6) and investigating the use of further simplified vehicle models involving simpler reachability analysis problems (see Remark 4).

We finally highlight that, as observed in Remark 7, the proposed threat assessment methods can be a convenient basis of decision making algorithms which blend driver's commands and supporting lower level autonomous driving interventions.

\section{ACKNOWLEDGEMENTS}

This work is partially supported by SAFER and VINNOVA, through the project "Systems for Roadway Departure Avoidance".

\section{REFERENCES}

[1] M. Althoff, O. Stursberg, and M. Buss. Model-based probabilistic collision detection in autonomous driving. IEEE Transactions on Intelligent Transportation Systems, 10(2):199-310, June 2009.

[2] Sterling J Anderson, Steven C Peters, Tom E Pilutti, and Karl Iagnemma. An optimal-control-based framework for trajectory planning, threat assessment, and semiautonomous control of passenger vehicles in hazard avoidance scenarios. Int. J. Vehicle Autonomous Systems, 8:190-216, 2010.

[3] C. Baker and J. Dolan. Traffic interaction in the urban challenge: Putting boss on its best behavior. International Conference on Intelligent Robots and Systems (IROS 2008), pages 569-597, September 2008.

[4] E. Bakker, L. Nyborg, and H. Pacejka. Tyre Modeling for Use in Vehicle Dynamics Studies. SAE, Paper 870421, 1989.

[5] A. M. Bayen, I. M. Mitchell, and C. J. Tomlin. Aircraft autolander safety analysis through optimal control-based reach set computation. Journal of Guidance, Control and Dynamics, 30(1):68-77, January-February 2007.

[6] M. Bertozzi, A. Broggi, and A. Fascioli. Visionbased intelligent vehicles: State of the art and perspectives. Robotics and Autonomous Systems, 32(1):1-16, juli 2000.

[7] D. P. Bertsekas. Control of Uncertain Systems with a setmembership description of the uncertainty. PhD thesis, Electronic Systems Laboratory, MIT, 1971. 
[8] D. P. Bertsekas and I. B. Rhodes. On the minimax reachability of target sets and target tubes. Automatica, 7:233-247, 1971.

[9] F. Blanchini. Set invariance in control - a survey. Automatica, 35(11):1747-1768, November 1999.

[10] S.P. Boyd and L. Vandenberghe. Convex optimization. Cambridge Univ Pr, 2004.

[11] X. Dai, A. Kummert, S. B. Park, and D. Neisius. A warning algorithm for Lane Departure Warning system. 2009 IEEE Intelligent Vehicles Symposium, pages 431435, juni 2009.

[12] A. Eidehall, J. Pohl, and F. Gustafsson. Joint road geometry estimation and vehicle tracking. Control Engineering Practice, 15(12):1484-1494, 2007.

[13] P. Falcone, F. Borrelli, J. Asgari, H. E. Tseng, and D. Hrovat. Predictive Active Steering Control for Autonomous Vehicle Systems. IEEE Transactions on Control Systems Technology, 15(3), 2007.

[14] P. Falcone, F. Borrelli, H. E. Tseng, J. Asgari, and D. Hrovat. Integrated braking and steering model predictive control approach in autonomous vehicles. Fifth IFAC Symposium on Advances of Automotive Control, 2007.

[15] P. Grieder. Efficient Computation of Feedback Controllers for Constrained Systems. $\mathrm{PhD}$ thesis, Institut für Automatik, Swiss Federal Institute of Technology Zurich, 2004.

[16] J. Jansson. Collision avoidance theory with application to automotive collision mitigation. $\mathrm{PhD}$ thesis, ISY, Linköping University, 2005.

[17] S. M. Kay. Fundamentals of statistical signal processing: estimation theory. Prentice hall signal processing series, 1993.

[18] U. Kiencke and L. Nielsen. Automotive Control Systems. Springer, 2005.

[19] I. Kolmanovsky and E. G. Gilbert. Theory and computation of disturbance invariant sets for discrete-time linear systems. Mathematical Problems in Egineering, 4:317367, 1998.

[20] M. Kvasnica, P. Grieder, and M. Baotić. Multi-Parametric Toolbox (MPT), 2004.

[21] S. Mammar, S. Glaser, and M. Netto. Time to Line Crossing for Lane Departure Avoidance: A Theoretical Study and an Experimental Setting. IEEE Transactions on Intelligent Transportation Systems, 7:226-241, 2006.

[22] D. T. McRuer, R. W. Allen, D. H. Weir, and R. H. Klein. New results in driver steering control models. Human Factors, 19(4):381-397, August 1977.

[23] U. Mellinghoff, T. Breitling, R. Schöneburg, and H. Metzler. The Mercedes-Benz Experimental Safety Vehicle 2009. In Proc.International Technical Conference on the Enhanced Safety of Vehicles Conference (ESV), pages 1$11,2009$.

[24] M. Montemerlo, J. Becker, S. Bhat, H. Dahlkamp, D. Dolgov, S. Ettinger, D. Haehnel, T. Hilden, G. Hoffmann, B. Huhnke, D. Johnston, S. Klumpp, D. Langer, A. Levandowski, J. Levinson, J. Marciland, D. Orenstein, J. Paefgen, I. Penny, A. Petrovskaya, M. Pflueger,
G. Stanek, D. Stavens, A. Vogt, and S. Thrun. Junior: The stanford entry in the urban challenge. Journal of Field Robotics, pages 569-597, September 2008.

[25] H. Peng and M. Tomizuka. Preview Control for Vehicle Lateral Guidance in Highway Automation. Journal of Dynamic Systems, Measurement, and Control, 115:679687, 1993.

[26] R. Philippsen, S. Kolski, K. Macek, and R. Siegwart. Path planning, replanning, and execution for autonomous driving in urban and offroad environments. In Proc. of The Workshop on Planning, Perception and Navigation for Intelligent Vehicles (ICRA), 2007.

[27] R. Rajamani. Vehicle Dynamics and Control. Springer, 2006.

[28] R. D. Roland and T. B. Sheridan. Simulation study of the driver's control of sudden changes in previewed path. Technical report, MIT, Department of Mechanical Engineering, 1967.

[29] E. J. Rossetter and J. C. Gerdes. A Study of Lateral Vehicle Control Under a Virtual Force Framework. In Proc. International Symposium on Advanced Vehicle Control, 2002.

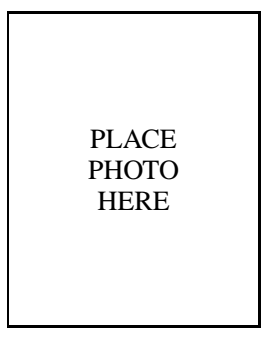

Paolo Falcone. Paolo Falcone received the "Laurea" degree in Computer Science Engineering in 2003 from the Universit di Napoli Federico II, Italy. In 2007 he received the $\mathrm{Ph}$. D. degree in Automatic Control from the Dipartimento di Ingegneria at Universitá del Sannio, Benevento, Italy.

Since April 2008 he is Assistant Professor in Mechatronics at the Department of Signals and Systems of the Chalmers University of Technology in Gteborg, Sweden.

His research interests include constrained optimal control, real-time model predictive control for fast automotive applications, vehicle dynamics control, active safety systems.

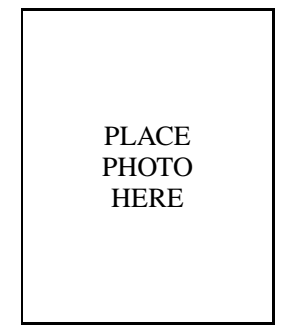

Mohammad Ali. Mohammad Ali received his M.S. degree in Electrical Engineering from Chalmers University of Technology.

Since 2007 he is pursuing the Ph.D degree in Mechatronics at Chalmers University of Technology. $\mathrm{He}$ is currently with the Active Safety and Chassis department at Volvo Car Corporation and his current research interests involve active safety and autonomous driving.

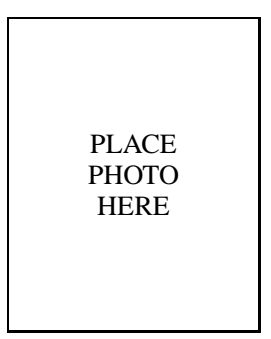

Jonas Sjöberg. Jonas Sjöberg, received the M.S. degree in engineering physics in 1989 from Uppsala University and the Ph. D. in 1995 from Linköping University, both in Sweden. He has held visiting research positions at ETH Zurich, Switzerland, TU Wien, Austria, Technion, Haifa, Israel, and Vrije Universiteit, Brussels, Belgium. He has served as an Associate Editor for Control and Control Engineering Practice (1999-2008), and he regularly serves as international program committee member at international conferences. He is Professor at the Department of Signals and Systems, Chalmers, Gothenburg, Sweden, since 2001. He is Programme Director of the Automation and Mechatronics education program. His research interests are in Mechatronics, and Mechatronic related fields, such as Signal Processing, and Control. Within these fields, interest focus on model based methods, simulations, system identification, and optimization for design and product development of Mechatronic systems. Current applications are, for example, Automotive Active Safety and Hybrid Electric Vehicles. 